\title{
Research on the Optical and EPR Spectral Data and the Local Structure for the Tetragonal $\mathrm{Nb}^{4+}$ Center in Glasses
}

\author{
Xiao-Xuan Wu ${ }^{a}$, Xin-Peng Yu ${ }^{a}$ And Wen-Chen Zheng ${ }^{b, *}$ \\ ${ }^{a}$ Department of Physics, Civil Aviation Flight University of China, Guanghan 618307, P.R. China \\ ${ }^{b}$ Department of Material Science, Sichuan University, Chengdu 610064, P.R. China
}

(Received November 29, 2014)

\begin{abstract}
Two optical bands and four EPR (or spin-Hamiltonian) parameters ( $g$ factors $g_{\|}, g_{\perp}$ and hyperfine structure constants $A_{\|}, A_{\perp}$ ) of the tetragonally-compressed $\mathrm{Nb}^{4+}$ octahedral center in glasses are calculated from two methods, the complete diagonalization (of energy matrix) method and the perturbation theory method. Both methods are founded on the two-spin-orbit-parameter model where both the contribution to the spectral data from the spin-orbit parameter of central $d^{n}$ ion and that of ligand ion via covalence effect are considered. The calculated results from both the complete diagonalization (of energy matrix) method and perturbation theory method show reasonable agreement with the experimental values. The signs and possible misprint for the observed hyperfine structure constants $A_{\|}$and $A_{\perp}$ are suggested, and the local structure of the tetragonal $\mathrm{Nb}^{4+}$ center in glasses due to the Jahn-Teller effect is gained from the calculations. The results are discussed.
\end{abstract}

DOI: 10.12693/APhysPolA.128.72

PACS: 76.30.He, 71.70.Ch, 78.40.Pg, 78.55.Qr

\section{Introduction}

Glasses doped with transition-metal $\left(d^{n}\right)$ and rareearth $\left(4 f^{n}\right)$ ions continue to be the attractive subject because of their widely potential applications in such as phosphor, luminescence, nonlinear optical and solid state laser materials [1-6] and microwave devices $[7,8]$. These applications are related to the oxidation states and structures of $d^{n}$ and $4 f^{n}$ impurity centers. Since the spectroscopic techniques can provide some information on the oxidation states and structures of these $d^{n}$ and $4 f^{n}$ impurity centers in glasses, many spectroscopic studies have been made for these doped glasses [9-14]. The optical and electron paramagnetic resonance (EPR) spectra of Nb-doped oxide glasses were measured [13]. The measurements suggested that there is a tetragonallycompressed $\mathrm{Nb}^{4+}$ octahedral center in these glasses with the ground state ${ }^{2} B_{2}\left(\left|d_{x, y}\right\rangle\right)$. The $g$ factors $g_{\|}, g_{\perp}$ and two optical bands assigned to $E_{1}\left({ }^{2} B_{2}-^{2} B_{1}\right)$ and $E_{2}$ $\left({ }^{2} B_{2}{ }^{2} E\right)$ were given for this $\mathrm{Nb}^{4+}$ center [13]. The EPR spectra of similar tetragonally-compressed $\mathrm{Nb}^{4+}$ octahedral center in glass with niobium oxide were also studied by Aleksandrov et al. [14]. The observed $g_{\|}$and $g_{\perp}$ of the $\mathrm{Nb}^{4+}$ center in Ref. [14] are very close to those in Ref. [13]. In addition, the observed hyperfine structure constants $A_{\|}$and $A_{\perp}$ were given in Ref. [14].

So far, the theoretical calculations for these optical and EPR spectral data of tetragonal $\mathrm{Nb}^{4+}$ center have not been undertaken. The theoretical calculations can confirm the oxidation state of $\mathrm{Nb}$ ion and gain the

${ }^{*}$ corresponding author; e-mail: zhengwc1@163.com information on the local structure of the $\mathrm{Nb}^{4+}$ center in glasses, so they are of interest. The optical and EPR spectral data of $d^{n}$ ions in materials can be calculated from two theoretical methods, the complete diagonalization (of energy matrix) method (CDM) and the perturbation theory method (PTM) [15-17]. The purpose of this paper is to calculate the optical and EPR spectral data of the tetragonal $\mathrm{Nb}^{4+}$ center in glasses from the $\mathrm{CDM}$ and PTM. Both methods are founded on the two-spinorbit-parameter model where not only the contributions due to the spin-orbit parameter of central $d^{n}$ ions in the conventional crystal-field theory, but also those of ligand ions via covalence effect are contained [18, 19]. The local structure of the tetragonal $\mathrm{Nb}^{4+}$ center due to the Jahn-Teller effect is also estimated from the calculations. The results are discussed.

\section{Two-spin-orbit-parameter model}

In the two-spin-orbit-parameter model, the oneelectron basis functions $\Psi(\gamma)$ of a $d^{n}$ octahedral cluster change from the pure $d$ orbitals $\left|d_{\gamma}\right\rangle$ of $d^{n}$ ion to the linear combinations of $\left|d_{\gamma}\right\rangle$ and $\left|p_{\gamma}\right\rangle$ (the $p$ orbitals of ligand ion), i.e., the molecular orbitals (MO) $[18,19]$ :

$$
\left|\Psi_{\gamma}\right\rangle=N_{\gamma}^{1 / 2}\left(\left|d_{\gamma}\right\rangle-\lambda_{\gamma}\left|p_{\gamma}\right\rangle\right)
$$

where $\gamma=\mathrm{t}$ or e denotes the irreducible representation of $O_{h}$ group. $N_{\gamma}$ and $\lambda_{\gamma}$ are the $\mathrm{MO}$ coefficients. Because of the basis functions, we have two spin-orbit parameters, two orbit reduction factors, and three dipolar hyperfine structure constants $P, P^{\prime}, P^{\prime \prime}[19]$ :

$$
\begin{aligned}
& \zeta=\zeta_{\mathrm{tt}}=N_{\mathrm{t}}\left(\zeta_{d}^{0}+\frac{1}{2} \lambda_{\mathrm{t}}^{2} \zeta_{p}^{0}\right), \\
& \zeta^{\prime}=\zeta_{\mathrm{te}}=\left(N_{\mathrm{t}} N_{\mathrm{e}}\right)^{\frac{1}{2}}\left(\zeta_{d}^{0}-\frac{1}{2} \lambda_{\mathrm{t}} \lambda_{\mathrm{e}} \zeta_{p}^{0}\right),
\end{aligned}
$$




$$
\begin{aligned}
& k=k_{\mathrm{tt}}=N_{\mathrm{t}}\left[1-2 \lambda_{\mathrm{t}} S_{d p}\left(t_{2 \mathrm{~g}}\right)+\frac{1}{2} \lambda_{\mathrm{t}}^{2}\right], \\
& k^{\prime}=k_{\mathrm{te}}=\left(N_{\mathrm{t}} N_{\mathrm{e}}\right)^{\frac{1}{2}}\left[1-2 \lambda_{\mathrm{t}} S_{d p}\left(t_{2 g}\right)\right. \\
& \left.\quad-\lambda_{\mathrm{e}} S_{d p}\left(e_{g}\right)-\frac{1}{2} \lambda_{\mathrm{t}} \lambda_{\mathrm{e}}\right], \quad P=P_{\mathrm{tt}}=N_{\mathrm{t}} P_{0}, \\
& P^{\prime}=P_{\mathrm{te}}=\left(N_{\mathrm{t}} N_{\mathrm{e}}\right)^{1 / 2} P_{0}, \quad P^{\prime \prime}=P_{\mathrm{ee}}=N_{\mathrm{e}} P_{0},
\end{aligned}
$$

in which the subscripts tt, te, and ee indicate the parameters related to the interactions within $t$ states, between $\mathrm{t}$ and e states and within e states, respectively. $\zeta_{d}^{0}$ and $P_{0}$ are the corresponding parameters of free $d^{n}$ ion and $\zeta_{p}^{0}$ is the spin-orbit parameter of free ligand ion. In the $\left(\mathrm{NbO}_{6}\right)^{8-}$ cluster under consideration, we have $\zeta_{d}^{0}\left(\mathrm{~N}_{\mathrm{b}}^{4+}\right) \approx 750 \mathrm{~cm}^{-1}[20], P_{0}\left(\mathrm{~N}_{\mathrm{b}}^{4+}\right) \approx$ $192 \times 10^{-4} \mathrm{~cm}^{-1}$ [21] and $\zeta_{p}^{0}\left(\mathrm{O}^{2-}\right) \approx 150 \mathrm{~cm}^{-1}[22,23]$. $S_{d p}(\gamma)$ are the group overlap integrals which can be calculated from the Slater-type self-consistent field (SCF) functions $[24,25]$ with the mean metal-ligand distance $R$. For $\left(\mathrm{NbO}_{6}\right)^{8-}$ cluster in glasses, we assume reasonably $R \approx 2.08 \AA$, the sum of ionic radii of $\mathrm{Nb}^{4+}$ and $\mathrm{O}^{2-}$ ions [26]. Thus, we have $S_{d p}(t) \approx 0.0301$ and $S_{d p}(e) \approx 0.0875$. The calculations of the parameters in Eq. (2) require the values of MO coefficients. These coefficients obey the normalization relationship

$$
N_{\gamma}\left[1-2 \lambda_{\gamma} S_{d p}(\gamma)+\lambda_{\gamma}^{2}\right]=1 \text {. }
$$

Thus, the coefficients $N_{\gamma}$ can be obtained if the coefficients $\lambda_{\gamma}$ are known. The coefficient $\lambda_{\mathrm{t}}$ being close to $\lambda_{\mathrm{e}}$ was found for $d^{n}$ octahedral clusters in materials [2729 , we therefore assume $\lambda_{\mathrm{t}} \approx \lambda_{\mathrm{e}} \approx \lambda_{\gamma}$ for decreasing the number of adjustable parameter and treat $\lambda_{\gamma}$ as an adjustable parameter in the following calculations.

\section{Calculation with CDM}

The Hamiltonian based on the two-spin-orbitparameter model for the calculations of optical and EPR spectral data of $d^{1}$ ions in tetragonal crystal field and under an external magnetic field (the demand of measuring the EPR parameters) can be expressed as

$$
\begin{aligned}
H & =H_{\mathrm{f}}+H_{\mathrm{SO}}\left(\zeta, \zeta^{\prime}\right)+H_{\mathrm{CF}}(D q, D s, D t) \\
& +H_{\mathrm{Ze}}\left(k, k^{\prime}\right)+H_{\mathrm{hf}}\left(P, P^{\prime}, P^{\prime \prime}\right)
\end{aligned}
$$

with the Zeeman (or magnetic) interaction term $H_{\mathrm{Ze}}$ and hyperfine interaction term $H_{\mathrm{hf}}$ [20]:

$$
\begin{aligned}
& H_{\mathrm{Ze}}=\mu_{\mathrm{B}}\left[\left(k, k^{\prime}\right) \boldsymbol{L}+g_{\mathrm{s}} \boldsymbol{S}\right] H_{\mathrm{M}}, \\
& H_{\mathrm{hf}}=\left(\boldsymbol{P}, \boldsymbol{P}^{\prime}, \boldsymbol{P}^{\prime \prime}\right)\left\{L+\left(\frac{4}{7}-\kappa\right) \boldsymbol{S}\right. \\
& \left.-\frac{1}{7}[(\boldsymbol{L} \cdot \boldsymbol{S}) \boldsymbol{L}+\boldsymbol{L}(\boldsymbol{L} \cdot \boldsymbol{S})]\right\} \cdot I,
\end{aligned}
$$

where $H_{\mathrm{f}}, H_{\mathrm{SO}}$ and $H_{\mathrm{CF}}$ are, respectively, the free ion, spin-orbit interaction and crystal field interaction (with the crystal field parameters $D q, D s$ and $D t$ ) terms. $g_{s} \approx$ 2.0023 is the free-ion $g$ value. $\kappa$ is the core polarization constant and the other symbols have their usual meanings $[20]$.

The $10 \times 10$ complete energy matrix of the Hamiltonian in Eq. (4) is established with the help of the strong field basis functions [30]. The eigenvalues of the energy matrix correspond to the crystal field energy levels and hence to the optical band positions. The EPR parameters can be calculated from the formulae

$$
\begin{aligned}
& g_{\|}=g_{z}=\frac{\Delta E_{\mathrm{Ze}}(z)}{\mu_{\mathrm{B}} H_{z}}, \quad g_{\perp}=g_{x}=\frac{\Delta E_{\mathrm{Ze}}(x)}{\mu_{\mathrm{B}} H_{z}}, \\
& A_{\|}=A_{z}=\Delta E_{\mathrm{hf}}(z), \quad A_{\perp}=A_{x}=\Delta E_{\mathrm{hf}}(x),
\end{aligned}
$$

in which $\Delta E_{\mathrm{Ze}}(j)(j=z$ or $x)$ stands for the Zeeman splitting under the external magnetic field along the $j$ direction and $\Delta E_{\mathrm{hf}}(j)$ denotes the hyperfine splitting with the operators in Eq. (5) along $j$ direction. They can be obtained by diagonalizing the energy matrix.

The crystal field parameters $D q, D s$, and $D t$ in energy matrix are calculated from the superposition model [31]. The model assumes that the crystal field parameters are the sum of contributions from the ligands and its reliability is supported by the angular overlap model [31] and the exchange charge model $[32,33]$. For the studied tetragonal system, the crystal field parameters in superposition model are given as

$$
\begin{aligned}
& D q=\frac{1}{21}\left(\frac{\sqrt{70}}{5}\right) B_{44}=\frac{4}{3} \bar{A}_{4}\left(R_{0}\right)\left(\frac{R_{0}}{R_{\perp}}\right)^{t_{4}}, \\
& D s=-\frac{1}{7} B_{20}=\frac{4}{7} \bar{A}_{2}\left(R_{0}\right)\left[\left(\frac{R_{0}}{R_{\perp}}\right)^{t_{2}}-\left(\frac{R_{0}}{R_{\|}}\right)^{t_{2}}\right], \\
& D t=-\frac{1}{21}\left(B_{20}-\frac{\sqrt{70}}{5} B_{44}\right)= \\
& \frac{16}{21} \bar{A}_{4}\left(R_{0}\right)\left[\left(\frac{R_{0}}{R_{\perp}}\right)^{t_{4}}-\left(\frac{R_{0}}{R_{\|}}\right)^{t_{4}}\right],
\end{aligned}
$$

where the power law exponents $t_{2} \approx 3$ and $t_{4} \approx 5[16$ $19,31]$ and $\bar{A}_{k}\left(R_{0}\right)(k=2,4)$ are the intrinsic parameters with the reference distance $R_{0}$ (which is often taken as the mean metal-ligand distance). For $4 d^{n}$ ions in materials, $\bar{A}_{2}\left(R_{0}\right) \approx(6 \pm 2) \bar{A}_{4}\left(R_{0}\right)$ was found [16, 34, 35] and here we take $\bar{A}_{2}\left(R_{0}\right) \approx 8 \bar{A}_{4}\left(R_{0}\right) . R_{\perp}$ and $R_{\|}$are the metal-ligand distances perpendicular to and parallel with the $C_{4}$ axis in the tetragonal $\left(\mathrm{NbO}_{6}\right)^{8-}$ octahedron. For $d^{1}$ ions in a regular octahedron, the ground state is the triply degenerate electron state ${ }^{2} T_{2}$. The degenerate ground state is unstable and will undergo a Jahn-Teller distortion to result in the change of octahedron from cubic to tetragonally-compressed, which can remove the degeneracy and lead the ground state to be undegenerate singlet ${ }^{2} B_{2}\left(\left|d_{x y}\right\rangle\right)$ (note that the octahedron cannot be changed as the tetragonally-elongated one because its ground state is still the unstable and degenerate doublet ${ }^{2} E$ ). So, we have

$$
R_{\perp}=R_{0}+\Delta R, \quad R_{\|}=R_{0}-2 \Delta R
$$

in which $\Delta R$ characterizes the tetragonal distortion due to the Jahn-Teller effect. Thus, in the energy matrix, there are only four parameters $\lambda_{\gamma}, \bar{A}_{4}\left(R_{0}\right), \Delta R$ and $\kappa$ left as the adjustable parameters. By matching the calculated optical and EPR data using CDM with the experimental values of the tetragonal $\mathrm{Nb}^{4+}$ center in glasses, we acquire 


$$
\begin{aligned}
& \lambda_{\gamma} \approx 0.54, \quad \bar{A}_{4}\left(R_{0}\right) \approx 2850 \mathrm{~cm}^{-1}, \\
& \Delta R \approx 0.155 \AA, \quad \kappa \approx 0.96 .
\end{aligned}
$$

The coefficients $N_{\gamma}$ acquired from coefficient $\lambda_{\gamma}$ are $N_{\mathrm{t}} \approx$ 0.7942 and $N_{\mathrm{e}} \approx 0.8354$. The parameters in Eq. (2) based on the MO coefficients are tabulated in Table I. The calculated optical and EPR spectral data are compared with the experimental values in Table II.

\section{TABLE I}

The spin-orbit parameters $\zeta, \zeta \prime\left(\right.$ in $\left.\mathrm{cm}^{-1}\right)$, the orbit reduction factors $k, k^{\prime}$ and the dipolar hyperfine structure constants $P, P^{\prime}$ and $P^{\prime \prime}$ (in $10^{-4} \mathrm{~cm}^{-1}$ ) of the $\left(\mathrm{NbO}_{6}\right)^{8-}$ octahedral cluster in glasses.

\begin{tabular}{c|c|c|c|c|c|c}
\hline \hline$\zeta$ & $\zeta \prime$ & $k$ & $k \prime$ & $P$ & $P \prime$ & $P \prime \prime$ \\
\hline 613 & 593 & 0.8842 & 0.6440 & 152 & 156 & 160
\end{tabular}

TABLE II

Optical and EPR spectral data for the tetragonally-

\begin{tabular}{|c|c|c|c|c|c|c|}
\hline & $E_{1}$ & $E_{2}$ & \multirow[t]{2}{*}{$g_{\|}$} & \multirow[t]{2}{*}{$g_{\perp}$} & $A_{\|}$ & $A_{\perp}$ \\
\hline & \multicolumn{2}{|c|}{$\left[\mathrm{cm}^{-1}\right]$} & & & \multicolumn{2}{|c|}{$\left[10^{-4} \mathrm{~cm}^{-1}\right.$} \\
\hline Calc. $^{a}$ & $\begin{array}{l}14884 \\
15502\end{array}$ & 26582 & 1.88 & 1.928 & -268 & -114 \\
\hline Calc. $^{b}$ & 15172 & 26528 & 1.88 & 1.928 & -264 & -116 \\
\hline Expt. [13] & $\begin{array}{c}16000 \\
\therefore \\
18000\end{array}$ & 26000 & 1.89 & 1.92 & & \\
\hline Expt. [14] & & & 1.88 & 1.91 & $262^{c}$ & $121^{c}$ \\
\hline
\end{tabular}
compressed $\left(\mathrm{NbO}_{6}\right)^{8-}$ cluster in glasses.

${ }^{a}$ Calculation with CDM.

${ }^{b}$ Calculation with PTM.

${ }^{c}$ The values are actually the absolute values.

\section{Calculations with PTM}

From the perturbation theory, the high-order perturbation formulae of EPR parameters founded on the twospin-orbit-parameter model for $d^{1}$ ion in tetragonallycompressed octahedron with the ground state ${ }^{2} B_{2}$ are derived as [18]:

$$
\begin{aligned}
g_{\|} & =g_{\mathrm{s}}-8 k^{\prime} \zeta^{\prime} / E_{2}-k \zeta^{2} /\left(E_{1}\right)^{2}-4 k^{\prime} \zeta \zeta^{\prime} /\left(E_{1} E_{2}\right) \\
& -g_{\mathrm{s}} \zeta^{2} /\left(E_{1}\right)^{2}, \\
g_{\perp} & =g_{\mathrm{s}}-2 k \zeta / E_{1}+k \zeta^{2} /\left(E_{1}\right)^{2}-2 k \zeta^{\prime 2} /\left(E_{1} E_{2}\right) \\
& +2 k^{\prime} \zeta \zeta^{\prime} /\left(E_{1} E_{2}\right)-2 g_{\mathrm{s}} \zeta^{\prime 2} /\left(E_{2}\right)^{2}-g_{\mathrm{s}} \zeta^{2} / 2\left(E_{1}\right)^{2}, \\
A_{\|} & =P(-\kappa-4 / 7)+P^{\prime}\left[-8 k^{\prime} \zeta^{\prime} / E_{2}-6 k \zeta /\left(7 E_{1}\right)\right. \\
& -4 k \zeta^{2} /\left(7 E_{1}\right)^{2}-6 k \zeta^{\prime 2} /\left(7 E_{1} E_{2}\right)-22 k^{\prime} \zeta \zeta^{\prime} /\left(7 E_{1} E_{2}\right) \\
& \left.-6 g_{\mathrm{s}} \zeta^{\prime 2} /\left(7 E_{2}\right)^{2}-17 g_{\mathrm{s}} \zeta^{2} /\left(14 E_{1}\right)^{2}\right], \\
A_{\perp} & =P(2 / 7-\kappa)+P^{\prime}\left[-11 k \zeta /\left(7 E_{1}\right)+11 / 14 k \zeta^{2} /\left(E_{1}\right)^{2}\right. \\
& -11 k \zeta^{\prime 2} /\left(7 E_{1} E_{2}\right)+11 k^{\prime} \zeta \zeta^{\prime} /\left(7 E_{1} E_{2}\right)
\end{aligned}
$$

$$
\left.-11 / 7 g_{\mathrm{s}} \zeta^{\prime 2} /\left(E_{2}\right)^{2}-11 / 28 g_{\mathrm{s}} \zeta^{2} /\left(E_{1}\right)^{2}\right]
$$

where the crystal field energy levels

$$
\begin{aligned}
& E_{1}=\Delta E\left({ }^{2} E-{ }^{2} B_{2}\right)=-3 D s+5 D t, \\
& E_{2}=\Delta E\left({ }^{2} B_{1}-{ }^{2} B_{2}\right)=10 D q .
\end{aligned}
$$

Substituting the same parameters used in the CDM into the above formulae, the optical and EPR spectral data of the tetragonally-compressed $\mathrm{Nb}^{4+}$ center in glasses are calculated with PTM. The results are also compared with the experimental values in Table II.

\section{Discussions}

The required core polarization constant $\kappa$ for fitting the observed hyperfine structure constants $A_{\|}$and $A_{\perp}$ is 0.96 . The large value of $\kappa(\approx 1)$ for $\mathrm{Nb}^{4+}$ ion is also found in $\mathrm{LiNbO}_{3}: \mathrm{Nb}^{4+}[35]$. So, it is advisable.

Only the EPR experiment is hard to determine the signs of hyperfine structure constants $A_{j}$ for $d^{n}$ and $4 f^{n}$ ions in materials $[20,21,36]$. So, even if the constants $A_{j}$ in these systems (including $\mathrm{Nb}^{4+}$ in glasses under study) are written as positive, they are actually the absolute values. For $d^{1}$ tetragonal octahedra with the ground state ${ }^{2} B_{2}$, the signs of $A_{j}$ are often opposite to that of the dipolar hyperfine constant $P_{0}$. For example, the constants $A_{j}>0$ for $\mathrm{Ti}^{3+}\left(P_{0} \approx-25.7 \times 10^{-4} \mathrm{~cm}^{-1}[21]\right)$, $\mathrm{Cr}^{5+}\left(P_{0} \approx-50.2 \times 10^{-4} \mathrm{~cm}^{-1}[21]\right)$ and $\mathrm{Mo}^{5+}\left(P_{0} \approx\right.$ $\left.-68.2 \times 10^{-4} \mathrm{~cm}^{-1}[21]\right)$, and $A_{j}<0$ for $\mathrm{V}^{4+}\left(P_{0} \approx\right.$ $\left.172 \times 10^{-4} \mathrm{~cm}^{-1}[21]\right), \mathrm{Nb}^{4+}\left(P_{0} \approx 192 \times 10^{-4} \mathrm{~cm}^{-1}[21]\right)$ and $\mathrm{W}^{5+}\left(P_{0} \approx 76 \times 10^{-4} \mathrm{~cm}^{-1}[34]\right)$ in materials were found $[18,21,34]$. So, for $\mathrm{Nb}^{4+}$ in glasses, the hyperfine structure constants $A_{j}$ should be negative, as suggested by our calculations (see Table II). More importantly, for $d^{1}$ tetragonal octahedral clusters with the ground state ${ }^{2} B_{2}$, the value of $\left|A_{\|}\right|$being greater than that of $\left|A_{\perp}\right|$ have been shown in many papers $[9,11,12$, 21,34 . So, there may be misprints in Ref. [14], i.e., the absolute values of $A_{\|}$and $A_{\perp}$ in [14] should be exchanged. Thus, the calculated $A_{\|}$and $A_{\perp}$ with the above advisable parameter $\kappa$ show reasonable agreement with the experimental values.

The tetragonal distortion parameter $\Delta R$ due to the Jahn-Teller effect is estimated from the calculations. So, some information on the local structure of paramagnetic impurity centers in materials can be gained by analyzing their optical and EPR spectral data.

Table II indicates that the six calculated optical and EPR data from CDM and PTM based on the two-spinorbit-parameter model for the tetragonally-compressed $\mathrm{Nb}^{4+}$ octahedral center in glasses are in reasonable agreement with the experimental values. The small disparities of spectral data between calculation and experiment are comprehensible because the vibrational contribution due to electron-phonon interaction to optical and EPR spectral data are ignored in the calculations. So, both CDM and PTM are valid in explaining the optical and EPR spectral data of $d^{1}$ ions in materials. 


\section{References}

[1] I.M. Thoms, S.A. Payne, G.D. Wilke, J. Non-Cryst. Solids 151, 183 (1992).

[2] M. Morita, N. Miyazaki, S. Murkami, M. Herren, D. Rau, J. Lumin. 76/77, 238 (1998).

[3] D.C. Lai, B. Dunn, J.I. Zink, Inorg. Chem. 35, 2152 (1996).

[4] M. Herren, H. Nishiuchi, M. Morita, J. Chem. Phys. 101, 4461 (1994).

[5] S.H. Kim, T. Yoko, J. Am. Ceram. Soc. 78, 1061 (1995).

[6] R.R. Rakhimov, D.E. Jones, H.L. Rocha, A.I. Prokofev, A.I. Aleksandrov, J. Phys. Chem. 104, 10973 (2000).

[7] T. Takada, S.F. Wang, S. Yoshikawa, S.J. Jang, R.E. Newhnam, J. Am. Ceram. Soc. 77, 1909 (1994).

[8] R.R. Rakhimov, D.E. Jones, H.L. Rocha, A.I. Prokofev, A.I. Aleksandrov, J. Phys. Chem. 104, 10973 (2000).

[9] A. Bals, J. Kliava, J. Phys. Condens. Matter 3, 6209 (1991).

[10] N.S. Rao, P.R. Rao, Y. Gandhi, Ch.S. Rao, G.S. Baskaran, V.R. Kumar, N. Veeraiah, Physica B 406, 4494 (2011).

[11] O. Cozar, I. Ardelean, J. Optoelectron. Adv. Matter 5, 177 (2003).

[12] O. Cozar, D.A. Magdas, I. Ardelean, J. Optoelectron. Adv. Matter 9, 1730 (2007).

[13] A. Goldstein, V. Chiriac, D. Becherescu, J. NonCryst. Solids 92, 271 (1987).

[14] A.I. Aleksandrov, N.N. Bubnov, A.I. Prokofev, Appl. Magn. Reson. 9, 251 (1995).

[15] C. Rudowicz, Y.Y. Yeung, Z.Y. Yang, J. Qin, J. Phys. Condens. Matter 14, 5619 (2002).

[16] W.C. Zheng, W. Fang, Y. Mei, J. Appl. Phys. 101, 053911 (2007).

[17] W.Q. Yang, W.C. Zheng, L. He, H.G. Liu, Spectrochim. Acta A 75, 458 (2010).
[18] W.C. Zheng, Y.J. Fan, X.X. Wu, Z. Naturforsch. A 60, 433 (2005).

[19] Y. Mei, W.C. Zheng, L. Zhang, Opt. Mater. 36, 535 (2013).

[20] A. Abragam, B. Bleaney, Electron Paramagnetic Resonance of Transition Ions, Oxford University Press, London 1970.

[21] B.R. McGarvey, J. Phys. Chem. 71, 51 (1967).

[22] M.L. Du, C. Rudowicz, Phys. Rev. B 46, 8974 (1992).

[23] W.C. Zheng, H.G. Liu, G.M. Jia, L. He, Spectrochim. Acta A 71, 1551 (2008).

[24] E. Clementi, D.L. Raimondi, J. Chem. Phys. 38, 2686 (1963).

[25] E. Clementi, D.L. Raimondi, W.P. Reinhardt, J. Chem. Phys. 47, 1300 (1967).

[26] C.R. Weast, CRC Handbook of Chemistry and Physics, CRC Press, Roca Raton, FL 1989, p. F-187.

[27] J.J. Chen, M.L. Du, J. Qin, Phys. Status Solidi B 174, K15 (1992).

[28] M.L. Du, M.G. Zhao, Phys. Status Solidi B 153, 249 (1989).

[29] M.L. Du, Phys. Rev. B 46, 5274 (1992).

[30] S. Sugano, Y. Tanabe, H. Kamimuru, Multiplets of Transition Metal Ions in Crystals, Academic Press, New York 1970.

[31] D.J. Newman, B. Ng, Rep. Prog. Phys. 52, 699 (1989).

[32] C. Rudowicz, M.G. Brik, N.M. Avram, Y.Y. Yeung, P. Gnutek, J. Phys. Condens. Matter 18, 5221 (2006).

[33] M.G. Brik, Y.Y. Yeung, J. Phys. Chem. Solids 69, 2401 (2008).

[34] W.C. Zheng, Y. Mei, W.Q. Yang, Philos. Mag. 89, 1621 (2009).

[35] W.C. Zheng, W. Fang, L. He, Y. Mei, J. Alloys Comp. 453, 32 (2009).

[36] V. Havliek, P. Novak, B.V. Mill, Phys. Status Solidi B 64, K19 (1974). 